\title{
Light-trapping in the near field: the case for plasmonic thin-film solar cells
}

\author{
A. Naqavi ${ }^{1,2}$, K. Söderström ${ }^{1}$, C. Pahud ${ }^{1}$, G. D. Osowiecki ${ }^{2}$, F.-J. Haug ${ }^{1}$, T. Scharf ${ }^{2}$, \\ H. P. Herzig ${ }^{2}$ and C. Ballif ${ }^{1}$ \\ ${ }^{1}$ Photovoltaics and Thin Film Electronics Laboratory, École Polytechnique Fédérale de Lausanne (EPFL), A. L. \\ Breguet 2, 2000 Neuchâtel, Switzerland \\ ${ }^{2}$ Optics \& Photonics Technology Laboratory, École Polytechnique Fédérale de Lausanne (EPFL), A. L. Breguet 2, \\ 2000 Neuchâtel, Switzerland \\ ali.naqavi@epfl.ch
}

\begin{abstract}
We study the plasmonic and the non-plasmonic regimes of operation of a thin-film amorphous-silicon solar cell. By rigorous calculation, we discuss the impact of the cell geometry on the nature of its optical resonances.
\end{abstract}

(C) 2013 Optical Society of America

OCIS codes: $050.0050,250.5403,040.5350$.

Thin-film silicon ( $\mathrm{Si}$ ) solar cells have opened an avenue towards responding the ever-increasing global energy demand in a sustainable economical way. Specifically amorphous $\mathrm{Si}$ (a-Si) cells have attracted attention because of their advantages such as low-cost fabrication and the possibility of low-temperature deposition on flexible plastic substrates. Thickness of the active Si layer in an a-Si cell is limited to around $300 \mathrm{~nm}$ because of light induced degradation. This implies weak light absorption in an a-Si cell at long-wavelength visible range, where the solar spectrum is most profitable. To overcome this challenge, different methods have been used to increase absorption in a-Si cells. Apart from putting a back-reflector underneath the cell, a cell's optical guided modes have been exploited to increase absorption in it [1]. Thanks to the compatibility of a-Si cells with plasmonics, recently different groups have focused on design and fabrication of plasmonic a-Si solar cells [2-4].

A thin-film a-Si solar cell normally consists of a p-i-n junction as its core, and two electrodes at the two sides of this junction to collect the photo-generated carriers. As depicted in Fig. 1(a), usually the back-electrode consists of a transparent conductive oxide (TCO) buffer layer and the back-reflector which can be made of Ag.

Here we study the effect of the buffer layer on the optical properties of the solar cell in detail by using rigorous electromagnetic calculations. It has been shown previously that the buffer layer modifies the nature of the cell's optical modes dramatically [5]. Here we investigate the dependence of plasmonic or guided modes in the cell on the thickness of this buffer layer. Also, we compare the application of $\mathrm{ZnO}$ or $\mathrm{MgF}_{2}$ as the buffer layer material. Furthermore, we discuss the conditions for the occurrence of plasmonic modes and the criteria to achieve better light-trapping in thin film solar cells by using either plasmonic or non-plasmonic modes.

As a first example, consider a cell with the following sequence of layers from top to bottom: ITO $(65 \mathrm{~nm})$, p-doped $\mathrm{Si}(15 \mathrm{~nm})$, intrinsic $\mathrm{Si}(250 \mathrm{~nm})$, n-doped $\mathrm{Si}(20 \mathrm{~nm}), \mathrm{ZnO}$ (variable thickness) and Ag. The optical band diagram of the planar device is obtained with the transfer matrix method in both TE and TM polarizations and is depicted in Fig. 1(b) and (c) for the $\mathrm{ZnO}$ buffer thicknesses of 0,10 and $70 \mathrm{~nm}$. The TE/TM polarization is the case where the electric/magnetic field is parallel to the interfaces. Changing $\mathrm{ZnO}$ thickness does not modify the bands in TE polarization significantly, but in TM polarization, it shifts lower order bands remarkably. Specifically, the lowest order TM mode $\left(\mathrm{TM}_{0}\right)$ goes underneath the light line of $\mathrm{Si}$ in the dispersion diagram for very thin $\mathrm{ZnO}$ layers, which is attributed to the existence of a plasmonic mode. Fig. 1(d) shows the normalized profile of the magnitude of magnetic field for the TM modes of the cell without $\mathrm{ZnO}$ buffer along the cell thickness at $700 \mathrm{~nm}$ wavelength $(1.77 \mathrm{eV})$. The $\mathrm{TM}_{0}$ mode is localized near the metallic interface which confirms its being plasmonic. Contrarily, the other modes are non-plasmonic guided modes. One can thus conclude that surface plasmons are present only when the buffer layer is very thin, in the range of a few nanometers.

As a second example, we study the change of buffer material. Fig. 1(e) shows the bands in TM polarization for a $\mathrm{ZnO}$ and $\mathrm{MgF}_{2}$ buffer layer. Thickness of the buffer layer is $70 \mathrm{~nm}$ in both cases. The TE bands are very similar and are not plotted here. $\mathrm{ZnO}$ has a larger refractive index compared to $\mathrm{MgF}_{2}$, which agrees with the blueshift of the TM 
bands for $\mathrm{MgF}_{2}$. The strongly confined $\mathrm{TM}_{0}$ and $\mathrm{TM}_{1}$ modes are very similar; differences appear for weakly confined modes that probe more of the outer material.

We continue to investigate the effect of the buffer layer material on the optical properties of a cell by considering a one-dimensional (1D) blazed grating Ag back-reflector for the cells in the previous example. The grating pattern is shown in the inset of Fig. 1(f) and the cells layers are conformal. By using the rigorous coupled-wave analysis (RCWA) method, we obtain the short-circuit current density $\left(J_{s c}\right)$ of the cell in both TE and TM polarization under different incidence angles. Incidence plane is normal to the grating grooves and TE/TM polarization is the case where the electric/magnetic field is parallel to the grating grooves. Fig. 1(f) shows the unpolarized (average of TE and TM) $J_{s c}$ as a function of the incident angle. The $\mathrm{ZnO}$ buffer provides higher $J_{s c}$ regardless of the incident angle.
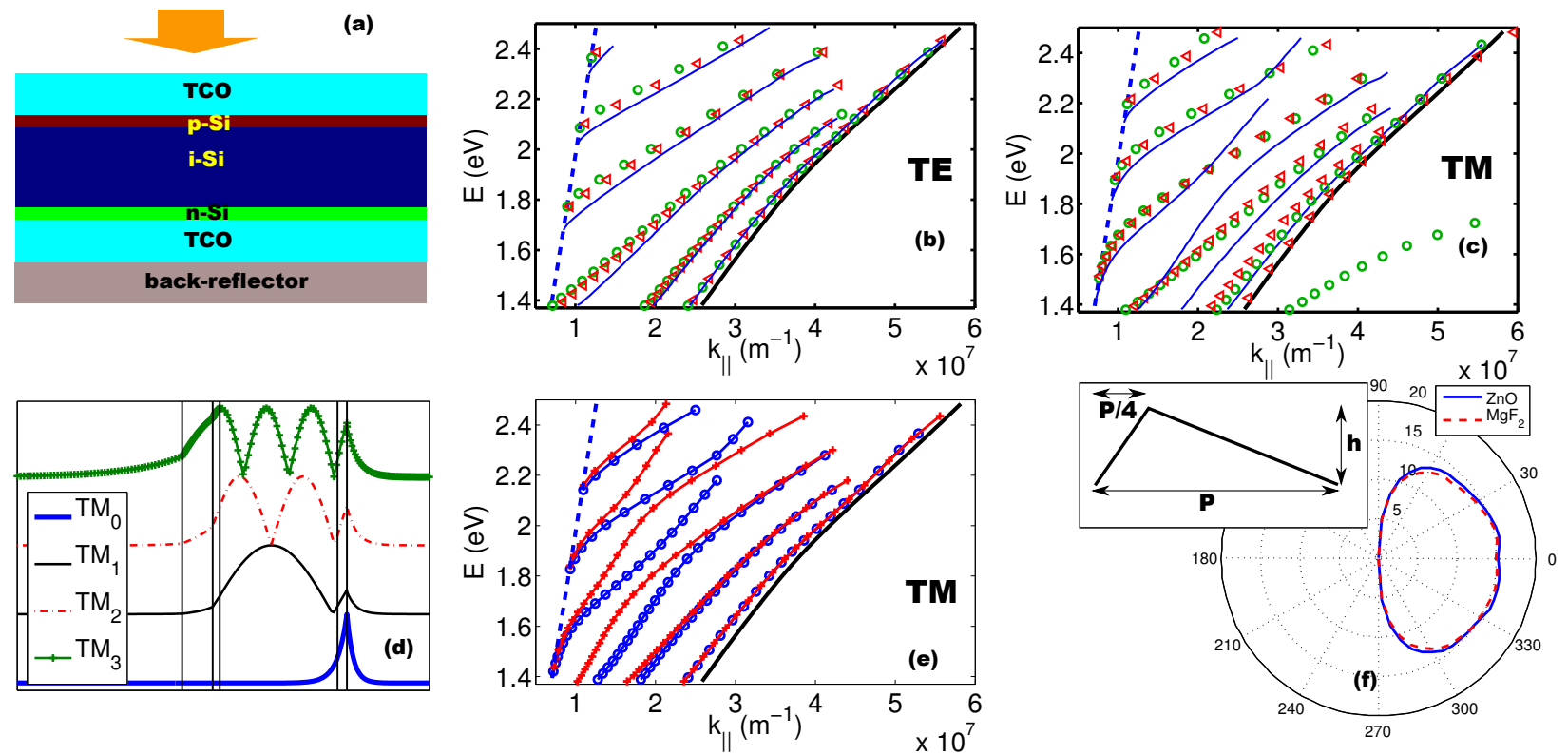

Fig. 1. (a): Schematic of the solar cell under investigation, (b): TE- polarized bands for the planar cell with different $\mathrm{ZnO}$ thicknesses: no $\mathrm{ZnO}$ (circles), $10 \mathrm{~nm}$ (triangles) and $70 \mathrm{~nm}$ (solid). The dashed and the black solid thick curves are the light lines of air and Si respectively. (c): same as (b) for TM polarization, (d): Profile of the TM modes plotted in (c) at $700 \mathrm{~nm}(1.77 \mathrm{eV})$ for the cell without buffer. Each profile is normalized to its peak value. The left and the right half spaces represent air and Ag. (e): TM-polarized bands of the cells with $70 \mathrm{~nm}$ of $\mathrm{ZnO}$ (circles) and $\mathrm{MgF}_{2}$ (curves with "+" symbols). (f): Angular behavior of $J_{s c}$ for the cells with $\mathrm{ZnO}$ and $\mathrm{MgF}_{2}$ buffer. The azimuth and the polar directions represent the incident angle (degrees) and the $J_{s c}$ $\left(\mathrm{mA} / \mathrm{cm}^{2}\right)$. The back-reflector is textured into the form of the 1D grating demonstrated in the inset. Grating period and height are $P=500 \mathrm{~nm}$ and $h=150 \mathrm{~nm}$ respectively.

This work was supported by Swiss National Science Foundation under project number 200021_125177/1.

\section{References}

1. K. Söderström, F.-J. Haug, J. Escarré, O. Cubero, and C. Ballif, "Photocurrent increase in n-i-p thin film silicon solar cells by guided mode excitation via grating coupler," Appl. Phys. Lett 96, 213508-213508 (2010).

2. H. A. Atwater, and A. Polman, "Plasmonics for improved photovoltaic devices," Nat. Mat. 9, 205-213 (2010).

3. H. Tan, R. Santbergen,A. H. M. Smets, and M. Zeman, "Plasmonic light trapping in thin-film silicon solar cells with improved self-assembled silver nanoparticles," Nano. Lett. 12, 4070-4076 (2012).

4. C. Pahud, O. Isabella, A. Naqavi, F.-J. Haug, M. Zeman, H. P. Herzig, and C. Ballif, "Plasmonic silicon solar cells: impact of material quality and geometry," Opt. Express 21, A786-A797 (2013).

5. F.-J. Haug,T. Söderström, O. Cubero, V. Terrazzoni-Daudrix, and C. Ballif, "Influence of the ZnO buffer on the guided mode structure in Si/ZnO/Ag multilayers," J. Appl. Phys. 106, 044502-044502 (2009). 